\title{
OSSC1E-K19, a novel phytochemical component of Osteomeles schwerinae, prevents glycated albumin-induced retinal vascular injury in rats
}

\author{
CHAN-SIK KIM ${ }^{1}$, JUNGHYUN KIM ${ }^{1}$, KYUHYUNG JO ${ }^{1}$, YUN MI LEE ${ }^{1}$, \\ EUNJIN SOHN $^{1}$, NAM HEE YOO ${ }^{2}$ and JIN SOOK KIM ${ }^{1}$
}

\author{
${ }^{1}$ Korean Medicine Based Herbal Drug Development Group, Herbal Medicine Research Division, \\ Korea Institute of Oriental Medicine, Daejeon 305-811; ${ }^{2}$ Korea Natural Products Development Laboratory, \\ International Ginseng and Herb Research Institute, Geumsan, South Chungcheong 312-804, Republic of Korea
}

Received December 4, 2014; Accepted August 24, 2015

DOI: $10.3892 / \mathrm{mmr} .2015 .4413$

\begin{abstract}
In the pathophysiology of diabetic retinopathy (DR), advanced glycation end products (AGEs) and vascular endothelial growth factor (VEGF) are thought to have important roles. It is known that VEGF causes a breakdown of the blood-retinal barrier (BRB) and retinal neovascularization; however, how AGEs affect the retina has largely remained elusive. OSSC1E-K19 is a novel phytochemical component of Osteomeles schwerinae. The objective of the present study was to evaluate the protective effects of OSSC1E-K19 on retinal vascular injury in AGE-modified rat serum albumin (AGE-RSA)-induced retinopathy. AGE-RSA-injected rat eyes were used investigate the protective effects of OSSC1E-K19 on BRB breakdown. Intravitreal injection of OSSC1E-K19 prevented AGE-RSA-induced BRB breakdown and decreased retinal VEGF expression in retinal vessels. In addition, OSSC1E-K19 inhibited the loss of occludin, a significant tight junction protein. These results supported the potential therapeutic utility of OSSC1E-K19 for retinal vascular permeability diseases.
\end{abstract}

\section{Introduction}

Diabetic retinopathy (DR), a vascular complication of diabetes mellitus, is characterized by blood-retinal barrier (BRB) breakage and subsequent retinal vascular permeability that represents a clinical hallmark of early DR. The integrity of

Correspondence to: Dr Jin Sook Kim, Korean Medicine Based Herbal Drug Development Group, Herbal Medicine Research Division, Korea Institute of Oriental Medicine, 1672 Yuseongdaero, Yuseong-gu, Daejeon 305-811, Republic of Korea

E-mail: jskim@kiom.re.kr

Key words: Osteomeles schwerinae, diabetic retinopathy, advanced glycation end products, vascular endothelial growth factor, blood-retinal barrier the BRB is required for normal vision and its breakage greatly contributes to the pathology of retinal diseases such as DR (1). The important mechanisms of BRB dysfunction are changes in the hyperpermeability characteristics of retinal endothelial cells (ECs) that are caused by changes in the internal environment of the retina; these changes include elevated levels of advanced glycation end products (AGEs), growth factors and hyperglycemia (2).

AGEs are thought to have a significant role in the course of DR by inducing BRB dysfunction (3). Although the detailed role of AGEs in retinal vascular permeability has remained elusive, previous studies have suggested a central role for vascular endothelial growth factor (VEGF) in this process $(4,5)$. Retinal VEGF expression was rapidly increased after a $4 \mathrm{~h}$ of exposure to AGE-modified bovine serum albumin (AGE-BSA) delivered via intravitreal (i.v.) injection (6). VEGF directly decreases the EC tight junction (TJ) content or increases their phosphorylation (7), either or the two of these effects may enhance paracellular permeability. In support of this, a previous study by our group has demonstrated that methylglyoxal, a potent precursor of AGEs, increased the expression of VEGF in retinal tissues, which elicits retinal vascular hyperpermeability (8).

Certain medicinal herbs that have been widely used for centuries for treating numerous human diseases. (4-hydroxy-3', 5'-dimetoxy-1,1'-biphenyl)-3-O- $\beta$-D-glucoside (OSSC1E-K19) is a novel biphenyl glycoside compound isolated from Osteomeles schwerinae C. K. Schneid. (Rosaceae). Osteomeles schwerinae (O. schwerinae) is a native plant of Asia and has been used as a Chinese medicinal herb for the treatments of numerous types of disease, including diarrhea, arthritis, dysentery, laryngopharyngitis, folliculitis and neuralgia (9). A previous study by our group showed that an ethanolic extract of this plant inhibited aldose reductase activity (10). OSSC1E-K19 was isolated from an ethyl acetate fraction of this plant using bioactivity-guided isolation. The present study was performed to investigate whether i.v. injection of OSSC1E-K19 was able to protect against glycated albumin-induced retinal vascular injury. In addition, the present study explored the underlying mechanisms of OSSC1E-K19 in rat eyes which were i.v. injected with glycated albumin. 


\section{Materials and methods}

OSSC1E-K19 preparation. The leaves and twigs of $O$. schwerinae were collected from Kunming, Yunnan Province, China, in April 2011, and identified by Professor Joo Hwan Kim (Gachon University, Korea). A voucher specimen (no. DiAB-141) was deposited in the Herbarium of the Diabetic Complications Research Team, Korea Institute of Oriental Medicine, Korea. Air-dried twigs and leaves of $O$. schwerinae (4 kg) were extracted with 121 ethanol $(\mathrm{EtOH})$ three times by maceration. The combined extracts were filtered and concentrated using a vacuum evaporator, and $104.16 \mathrm{~g}$ of the EtOH extract was obtained. The EtOH extract was suspended in $500 \mathrm{ml}$ $\mathrm{H}_{2} \mathrm{O}$. This $\mathrm{H}_{2} \mathrm{O}$ suspension was fractionated by liquid-liquid partitioning using n-hexane $(500 \mathrm{ml})$, EtOAc $(500 \mathrm{ml})$ and $\mathrm{n}-\mathrm{BuOH}(500 \mathrm{ml})$ to yield an $\mathrm{n}$-hexane fraction of $6.30 \mathrm{~g}$, an EtOAc fraction of $27.0 \mathrm{~g}$ and an $\mathrm{n}-\mathrm{BuOH}$ fraction of $10.08 \mathrm{~g}$. All of the factions were tested for AGE formation. The EtOAc fraction $(27 \mathrm{~g})$, which had the highest inhibitory effect on AGE formation, was loaded into a column $(7 \times 57 \mathrm{~cm})$ that was packed with 70-230 mesh silica gel. Silica gel (Merck Millipore, Darmstadt, Germany) chromatography was performed using a mobile phase of $\mathrm{CHCl}_{3}$ and $\mathrm{MeOH}$ (gradient of 40:1-0:1, $\mathrm{v} / \mathrm{v})$; ten fractions were obtained. The fifth fraction $(700 \mathrm{mg})$ was loaded onto a column $(60 \times 2.5 \mathrm{~cm})$ packed with Sephadex LH-20 gel (GE Healthcare Life Sciences, Chalfont, UK), and column chromatography was performed using a mobile phase of $\mathrm{MeOH}$ and distilled $\mathrm{H}_{2} \mathrm{O}$ (gradient of 1:1-1:0, v/v). A total of OSSC1E-K19 (10 mg; yield, 0.04\%) was isolated. The structure of OSSC1E-K19 was identified as 4-hydroxy -3',5'-dimethoxy-(1,1'-biphenyl)-3-O- $\beta$-D-glucopyranoside by comparing its nuclear magnetic resonance (NMR) and high resolution electrospray ionization mass (HRESIMS) data with those in the literature (11); $1 \mathrm{H}$ and 13C-NMR spectra were obtained using a Bruker Avance ${ }^{300}$ NMR spectrometer (Bruker AXS, Karlsruhe, Germany) with tetramethylsilane as an internal standard, and HRESIMS was recorded on a Shimadzu LCMS-IT-TOF spectrometer (Shimadzu Corporation, Kyoto, Japan). The chemical structure of OSSC1E-K19 is presented in Fig. 1.

Inhibitory activity on AGE formation. Ten milligrams per milliliter bovine serum albumin (BSA; Roche Diagnostics, Basel, Switzerland) in $50 \mathrm{mM}$ phosphate buffer (pH 7.4) containing $0.01 \%$ sodium azide (to avoid microbial contamination; cat. no. S-8032, Sigma-Aldrich, St. Louis, MO, USA) was added to a $0.2 \mathrm{M}$ glucose and fructose solution (Sigma-Aldrich); this solution was added to the samples (1-150 $\mu \mathrm{M})$. Aminoguanidine (AG; cat. no. 396494; Sigma-Aldrich) was used as a positive inhibitor. After incubation for 14 days at $37^{\circ} \mathrm{C}$, AGEs-specific fluorescence was determined using a spectrofluorometer (Synergy HT; BIO-TEK, Winooski, VT, USA; excitation at $370 \mathrm{~nm}$ and emission at $440 \mathrm{~nm}$ ). The concentration leading to $50 \%$ inhibition of AGE formation $\left(\mathrm{IC}_{50}\right)$ was determined.

Inhibitory activity on AGE cross-linking with collagen. AGE-BSA (Wako Pure Chemical Industries, Ltd., Osaka, Japan) was conjugated with horseradish peroxidase (HRP) using a peroxidase labeling kit-NH2 (Dojindo Molecular Technologies,

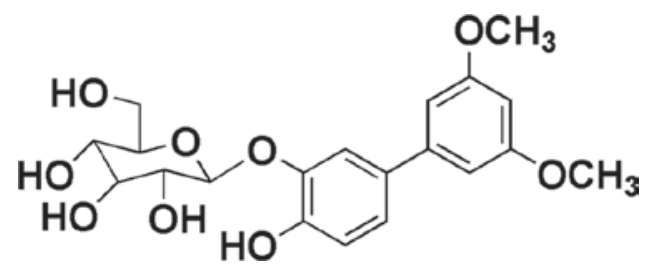

Figure 1. Chemical structure of OSSC1E-K19.

Inc., Kumamoto, Japan). HRP-labeled AGE-BSA was incubated with or without OSSC1E-K19 or AG in collagen-coated microtiter plates. HRP-labeled AGE-BSA was incubated to react with collagen at $37^{\circ} \mathrm{C}$ for $4 \mathrm{~h}$. After washing with a solution of $0.05 \%$ Triton-X 100 in phosphate-buffered saline (PBS), $100 \mu 1$ 3,3',5,5'-tetramethylbenzidine chromogen (cat. no. T4444; Sigma-Aldrich) was added followed by incubation for $15 \mathrm{~min}$. The absorbance at $450 \mathrm{~nm}$ was determined using an absorbance plate reader (Synergy HT) after the addition of $40 \mu \mathrm{l} 1 \mathrm{~N} \mathrm{H}_{2} \mathrm{SO}_{4}$.

Preparation of AGE-modified rat serum albumin (RSA). AGE-RSA was synthesized as previously described (12). Non-modified RSA (fraction V, low-endotoxin; Sigma-Aldrich) was used as the control. The components of AGE-RSA were determined using a glucose-derived AGE ELISA kit (Cosmo Bio, Tokyo, Japan). The modified levels of AGE-RSA were nearly 200-fold higher than those in the non-modified control. No endotoxins were detected using the E-Toxate kit (cat. no. ET0200-1KT; Sigma-Aldrich).

Intravitreal injection of AGE-RSA. Injection of AGE-RSA was performed as previously described (13). A total of 40 male eight week-old Sprague-Dawley rats (Orient Bio, Inc., Sungnam, Korea) were used in the present study. Rats were housed four per cage in a sterile humidified environment (50-60\%) at $23 \pm 1^{\circ} \mathrm{C}$, with a 12 -h light/dark cycle. Food and water were provided ad libitum throughout the experiment. The rats were divided into five groups: $6 \mu \mathrm{g}$ RSA-injected normal rats; rats injected with $6 \mu \mathrm{g}$ AGE-RSA i.v.; and rats injected with AGE-RSA i.v. and treated with different concentrations of OSSC1E-K19 $(50,100$ or $150 \mu \mathrm{M})$. For the negative control, $150 \mu \mathrm{M}$ OSSC1E-K19 was injected. Three days after i.v. injection, the rats were anesthetized and sacrificed with an overdose of zolazepam $(10 \mathrm{mg} / \mathrm{kg}$; Virbac, Carros, France). The present study was approved by the Institutional Animal Care and Use Committee of the Korea Institute of Oriental Medicine (protocol no. 12-079; Daejeon, Korea).

Fluorescein-dextran microscopy. The rats were deeply anesthetized by intraperitoneal injection of zolazepam $(10 \mathrm{mg} / \mathrm{kg}$; Virbac), after which PBS containing fluorescein-dextran (FD40S; Sigma-Aldrich) was injected into the left ventricle of the heart. The retinal flat was mounted, and the fluorescent images were captured using an Olympus BX51 microscope with a DP71 digital camera (Olympus, Tokyo, Japan).

Immunofluorescence staining. Retinal sections were de-waxed and re-hydrated by sequential immersion. The slides were boiled in $10 \mathrm{mM}$ sodium citrate buffer $(\mathrm{pH} 6.0)$ at $121^{\circ} \mathrm{C}$ for 
Table I. Inhibitory effects of OSSC1E-K19 and AG on AGE formation.

\begin{tabular}{lccc}
\hline Compound & Concentration $(\mu \mathrm{M})$ & Inhibition $(\%)$ & $\mathrm{IC}_{50}(\mu \mathrm{M})$ \\
\hline OSSC1E-K19 & 24.5 & $25.53 \pm 1.16$ & $118.49 \pm 4.85$ \\
& 49 & $37.67 \pm 2.40$ & $50.86 \pm 1.17$ \\
AG & 122.5 & $39.93 \pm 1.74$ & $1040.70 \pm 44.17$ \\
& 750 & $49.97 \pm 3.22$ & \\
& 1000 & $56.62 \pm 2.25$ & \\
\hline
\end{tabular}

Inhibitory effects are expressed as the mean \pm standard deviation of quadruplicate experiments. The $\mathrm{IC}_{50}$-value was calculated from the dose inhibition curve. AG was used as a positive control. AG, aminoguanidine; AGE, advanced glycation end product.
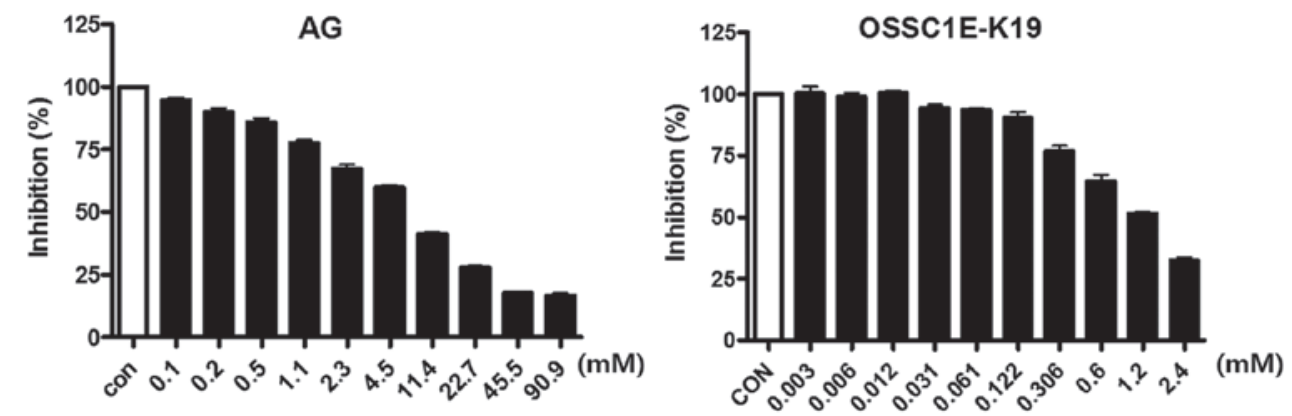

Figure 2. Inhibitory effects of OSSC1E-K19 on the cross-linking of AGEs to collagen in vitro. The levels of AGE-bovine serum albumin attached to collagen were determined by ELISA. Values are expressed as the mean \pm standard error of the mean $(\mathrm{n}=4)$. ${ }^{*} \mathrm{P}<0.05$ vs. CON. CON, control; AG, aminoguanidine; AGE, advanced glycation end product.
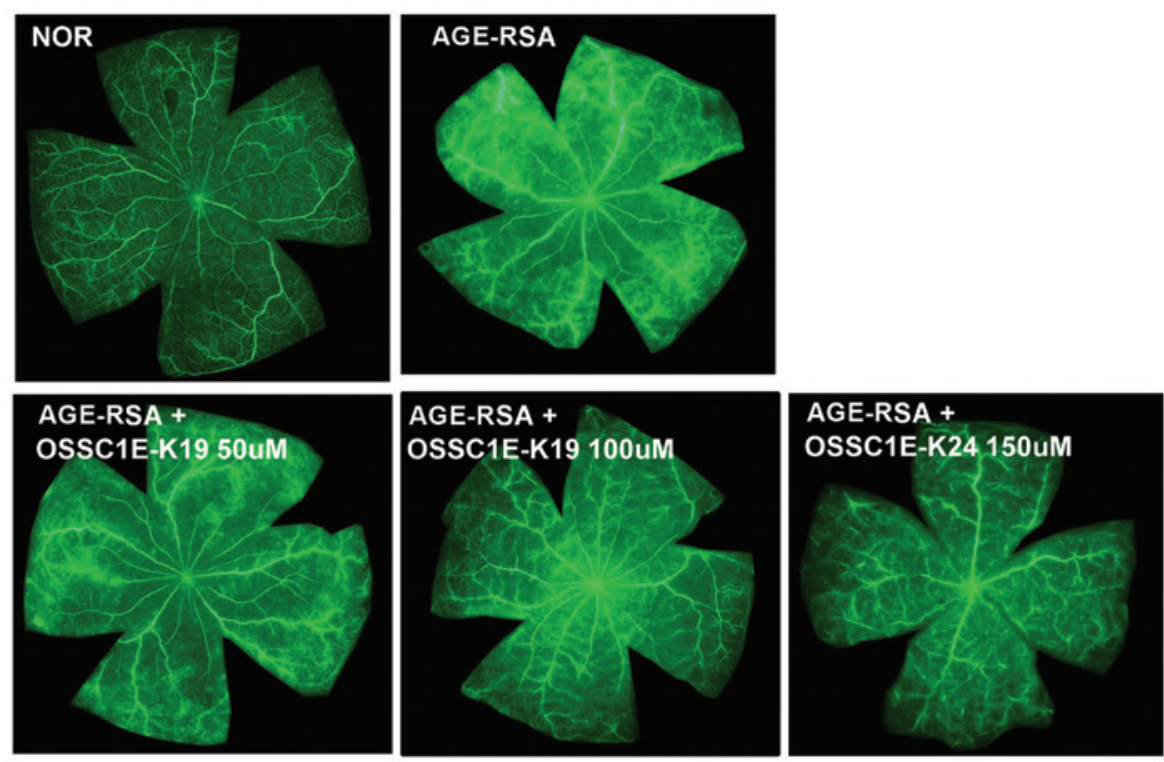

Figure 3. Fluorescein isothiocyanate-dextran angiography displaying retinal flat mounts to assess BRB in experimental groups as indicated. Normal retinas showed no leakage of the tracer into the retina, as is apparent by the clear delineation of the retinal capillaries. AGE-RSA retinas demonstrated a widespread breakdown of their BRB with tracer leakage into the neural retina and a loss of delineation of the retinal capillaries. However, treatment with OSSC1E-K19 significantly decreased retinal vascular permeability in the AGE-RSA-injected eye. Magnification, x12.5. Nor, saline-injected eye; AGE-RSA, advanced glycation end product-rat serum albumin; BRB, blood-retinal barrier breakdown.

$10 \mathrm{~min}$. The slides were incubated with a mouse anti-VEGF antibody (1:500; Santa Cruz Biotechnology, Inc., Dallas, TX, USA) for $2 \mathrm{~h}$. After washing, the sections were labeled with fluorescein isothiocyanate-conjugated donkey anti-mouse immunoglobulin G (1:3,000; Santa Cruz Biotechnology, Inc.) for $1 \mathrm{~h}$ at room temperature. Finally, the sections were mounted in fluorescent mounting medium (Dako North America, Inc., Carpinteria, CA, USA) containing DAPI counterstain. The 
A
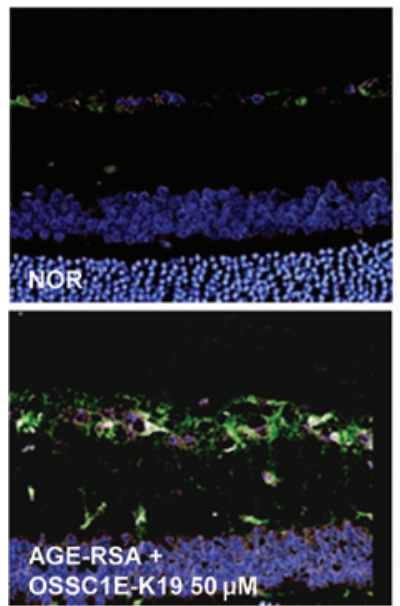
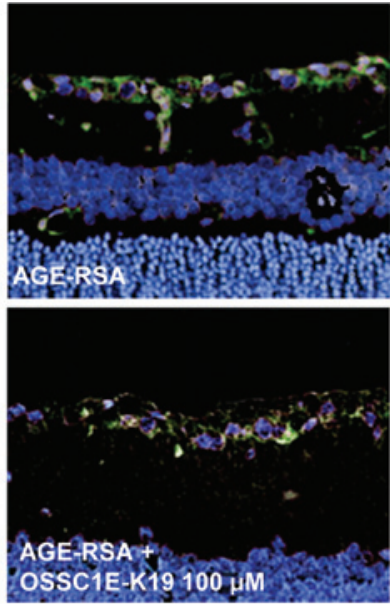

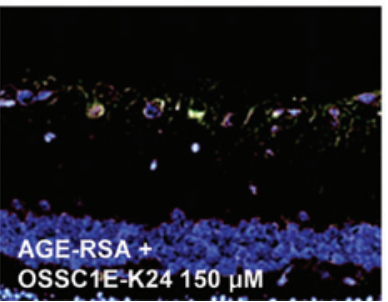

B
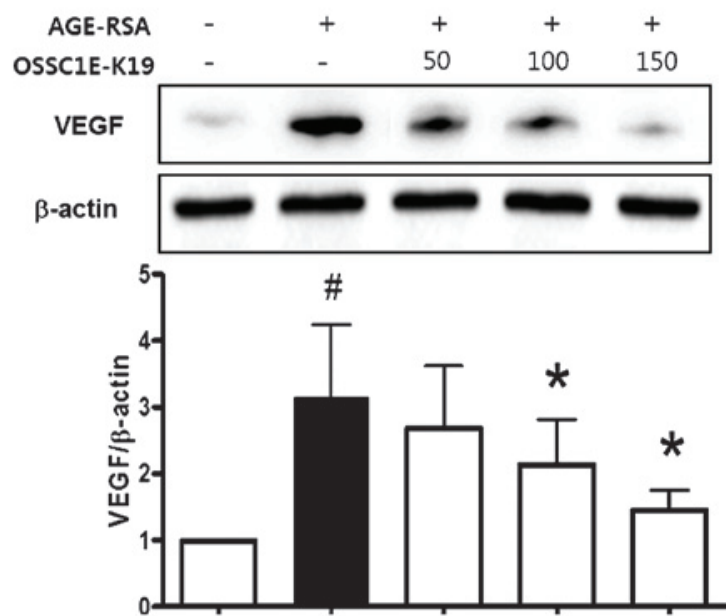

Figure 4. Effects of OSSC1E-K19 on the expression of VEGF in the retinas. (A) VEGF was determined by immunofluorescence staining (magnification, $\mathrm{x} 200$ ) and (B) western blot analysis followed by densitometric quantification. Values are expressed as the mean \pm standard error $(\mathrm{n}=8)$. ${ }^{*} \mathrm{P}<0.01$ vs. Nor group; ${ }^{*} \mathrm{P}<0.01$ vs. AGE-RSA-injected group. VEGF, vascular endothelial growth factor; Nor, saline-injected eye; AGE-RSA, advanced glycation end product-rat serum albumin.

sections were observed and images were captured by fluorescence microscopy (BX51; Olympus, Tokyo, Japan).

Western blot analysis. Retinas were homogenized (Precellys 24; Bertin Technologies, Saint-Quentin en Yveline, France) in solutions containing $250 \mathrm{mM}$ sucrose, $1 \mathrm{mM}$ ethylenediaminetetraacetic acid, $0.1 \mathrm{mM}$ phenylmethylsulfonyl fluoride (Sigma-Aldrich) and $20 \mathrm{mM}$ potassium phosphate buffer (Sigma-Aldrich; pH 7.6). Retinal protein $(20 \mu \mathrm{g})$ was resolved by $12 \%$ SDS-PAGE and transferred onto a polyvinylidene difluoride membrane (Bio-Rad Laboratories, Inc., Hercules, CA, USA) and the membranes were incubated with the following primary monoclonal antibodies overnight at $4^{\circ} \mathrm{C}$ : Antibodies against VEGF (1:1,000; cat. no. ab1316; Abcam, San Francisco, CA, USA) and occludin (1:1,000; cat. no. 711500; Invitrogen Life Technologies, Inc., Carlsbad, CA, USA) were used. Monoclonal anti- $\beta$-actin antibody (1:3,000; cat. no. A1978) was purchased from Sigma-Aldrich. Either goat anti-mouse IgG HRP (cat. no. sc-2005) or anti-rabbit IgG HRP-conjugated antibody (cat. no. sc-2004; 1:2,000; Santa Cruz Biotechnology, Inc.) was used as a secondary antibody. Immunoreactive bands were visualized using an enhanced chemiluminescence western blotting detection system (Amersham Bioscience, Amersham, NJ, USA). Images were captured and quantification was performed using a LAS-3000 (Fujifilm, Tokyo, Japan).

Statistical analysis. Values are expressed as the mean \pm standard error of the mean. Statistical analysis was performed using one-way analysis of variance followed by Tukey's multiple comparisons test or an unpaired Student's t-test. GraphPad Prism 6.0 (GraphPad Inc., La Jolla, CA, USA) was used for all statistical analyses. $\mathrm{P}<0.05$ was considered to indicate a statistically significant difference between values.

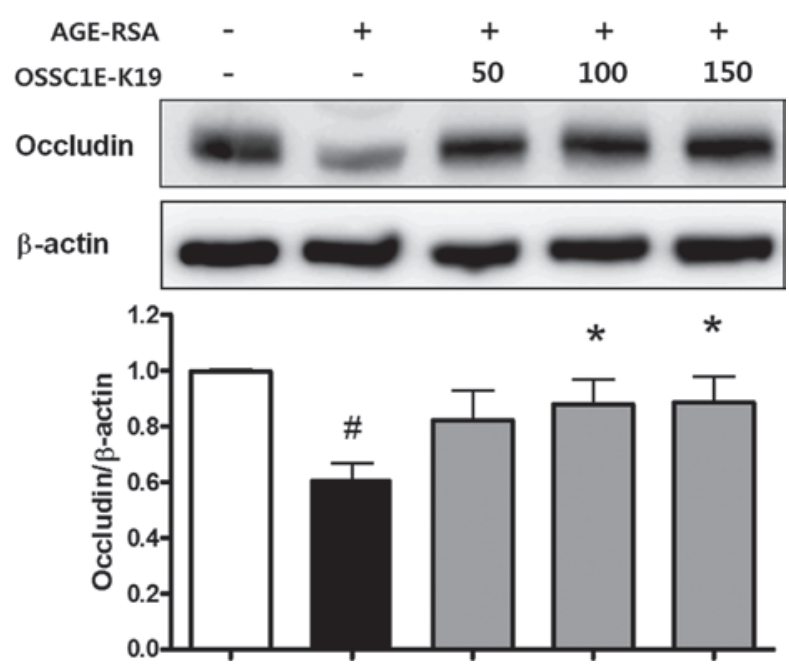

Figure 5. Effects of OSSC1E-K19 on AGE-RSA-induced occludin loss. Total protein was isolated for western blot analysis. Occludin expression in the AGE-RSA-injected retinas was lower than that in normal retinas. Values are expressed as the mean \pm standard error of the mean $(n=8)$. ${ }^{*} \mathrm{P}<0.01$ vs. normal group; ${ }^{~} \mathrm{P}<0.01$ vs. AGE-RSA-injected group. AGE-RSA, advanced glycation end product-rat serum albumin.

\section{Results}

Inhibitory effect of OSSC1E-K19 on AGEs formation and its cross-linking with collagen in vitro. OSSC1E-K19 was examined for its ability to inhibit AGE formation. As shown in Table I, OSSC1E-K19 dose-dependently inhibited the formation of AGE-BSA $\left(\mathrm{IC}_{50}, 118.49 \pm 4.85 \mu \mathrm{M}\right)$. The inhibitory activity of OSSC1E-K19 was greater than that of $\mathrm{AG}\left(\mathrm{IC}_{50}\right.$, $1,040.70 \pm 44.17 \mu \mathrm{M})$. In addition, OSSC1E-K19 dose-dependently inhibited the cross-linking of AGE-BSA with collagen, 
and the $\mathrm{IC}_{50}$ value of OSSC1E-K19 was $8.20 \pm 0.60 \mathrm{mM}$ (Fig. 2). AG also reduced cross-linking between AGE-BSA and collagen $\left(\mathrm{IC}_{50}, 1.39 \pm 0.13 \mathrm{mM}\right)$.

Effects of OSSC1E-K19 on BRB breakdown in $A G E-R S A$-injected rat eyes. A major clinical hallmark of DR is enhanced vascular leakage culminating in overt BRB breakage (14). To test the ability of OSSC1E-K19 to inhibit AGE-induced vascular permeability, the present study performed fluorescein angiography in rats that were injected with AGE-RSA i.v.. As shown in Fig. 3, the fluorescence intensity was enhanced, with dye leakage occurring throughout the entire retina in the AGE-RSA-injected eyes; however, these leaks were restricted to the vasculature in the saline-injected normal rat eyes and in OSSC1E-K19-injected eyes.

Effects of OSSC1E-K19 on retinal VEGF expression in $A G E-R S A$-injected rat eyes. The inhibitory effects of OSSC1E-K19onretinalVEGFexpressioninAGE-RSA-injected eyes were assessed by determining retinal VEGF expression using immunofluorescence staining and western blot analysis. In AGE-RSA-injected eyes, immunofluorescence staining for VEGF revealed that VEGF (green) was present in the ganglion cell layer, while treatment with OSSC1E-K19 significantly decreased retinal VEGF expression in AGE-RSA-injected eyes (Fig. 4A). These immunofluorescence staining results were confirmed by western blot analysis. As shown in Fig. 4B, AGE-RSA treatment led to a significant increase in VEGF expression compared with that in the saline-injected normal group. However, treatment with OSSC1E-K19 markedly inhibited the expression of VEGF in AGE-RSA-injected eyes. Similarly, treatment with OSSC1E-K19 almost completely eliminated the increase in retinal vascular permeability that was observed in AGE-RSA-injected eyes (Fig. 3). These results strongly suggested that OSSC1E-K19 inhibits retinal vascular permeability via the suppression of retinal VEGF expression.

Effects of OSSC1E-K19 on TJ protein loss in AGE-RSA-injected rat eyes. The loosening of TJs causes BRB breakdown (15). The present study evaluated the expression of occludin, a well-known TJ protein. AGE-RSA-injected eyes exhibited significantly lower levels of occludin than those of saline-injected normal mice. However, the decreased occludin expression in the AGE-RSA-injected eyes was restored by OSSC1E-K19 treatment (Fig. 5). These findings suggested that OSSC1E-K19 blocks vascular leakage, possibly by stabilizing junctional proteins.

\section{Discussion}

AGE-induced retinal damage is one of the most important mechanisms involved in the pathophysiology of DR. Numerous previous studies have demonstrated that hyperglycemia has an important role in the pathophysiology of DR by increasing protein glycation in ocular disease $(16,17)$. Cross-links between AGEs and long-lived proteins are formed when glucose binds to target proteins, including collagen, in a process that is nearly irreversible. Therefore, it is of great interest to identify novel, therapeutically useful inhibitory agents against AGE formation or AGE cross-links (18). Various synthetic and natural products have been tested and proposed as AGE inhibitors (19,20). AG, as the most well-known AGE inhibitor, attenuated diabetic angiogenesis, apoptosis and cataract formation in diabetic ocular disease $(17,21)$. The present study demonstrated that OSSC1E-K19 has an inhibitory effect on the formation of AGEs and their cross-linking with collagen in vitro.

In order to evaluate the potential therapeutic applicability of OSSC1E-K19 as an AGE inhibitor, the present study evaluated the efficacy of OSSC1E-K19 against BRB breakage induced by AGE-RSA in rats. AGE levels in the circulation are greatly enhanced in patients with diabetes (22). Previous studies have proven that i.v. injection of AGEs into the eyes of normal rats can induce a diabetic-like vascular injury $(23,24)$. The administration of exogenous AGEs induces acute vascular leakage in normoglycemic animals (24), whereas the inhibition of endogenous AGEs prevents vascular permeability in diabetic rats (25). Similarly, OSSC1E-K19, a potent AGE inhibitor, inhibited vascular hyperpermeability in AGE-RSA-injected eyes, as demonstrated in the present study.

A close correlation has been identified between AGE formation and VEGF expression in the diabetic retina $(4,5)$. AGEs can increase the expression of VEGF in retinal pigment epithelial cells, Müller cells and smooth muscle cells in vitro (6). Of note, Lu et al (6) demonstrated that VEGF was upregulated in the inner nuclear layer and ganglial cells following i.v. injection of AGE-BSA into rat retina. Consistent with these previous studies, the present study showed that i.v. injection of AGE-RSA stimulated the expression of VEGF in rat retina. OSSC1E-K19 decreased retinal VEGF expression in the AGE-RSA-injected eye. These results suggested that OSSC1E-K19 may prevent AGE-induced BRB breakage by inhibiting VEGF-associated signaling pathways.

VEGF promotes vascular permeability and alters TJs in retinal diseases. VEGF is thought to have a role in diabetic rats (7), and exposure of retinal ECs and the retina to exogenous VEGF reduced TJ protein contents and subsequently increased BRB breakage (26). AGE-BSA induces vascular leakage in a time- and concentration-dependent manner due to suppression of the expression of TJ proteins, including occludin and ZO-1 (27). TJs are composed of transmembrane adhesive proteins and scaffolding proteins, as well as a number of regulatory proteins that also localize to TJs. Occludin is an important transmembrane protein in TJs that is responsible for forming the permeability barrier (28). A number of studies have demonstrated a reduced expression of occludin in retinal ECs in diabetic animals $(29,30)$. Occludin was originally predicted to confer barrier properties to TJs (28) and gene deletion of occludin gave rise to mice with an array of complex phenotypes but with normal TJ formation and barrier properties in the gut epithelium (31). An inverse correlation between endothelial permeability and TJ protein content has been indicated. The present study observed that i.v. injection of OSSC1E-K19 resulted in a stabilization of the occludin content. These results suggested that OSSC1E-K19 reduced retinal vascular permeability in AGE-RSA-injected eyes via recovering occludin levels.

In conclusion, the present study demonstrated that OSSC1E-K19 has an inhibitory effect on the formation of AGEs and their cross-linking with proteins. In rats that were i.v. injected with AGE-RSA, treatment with OSSC1E-K19 
inhibited AGE-induced vascular hyperpermeability and resulted in the concomitant downregulation of VEGF and preservation of TJ integrity.

\section{Acknowledgements}

The present study was supported by a grant from the Korea Institute of Oriental Medicine (KIOM) (no. K14040).

\section{References}

1. Cheung N, Mitchell P and Wong TY: Diabetic retinopathy. Lancet 376: 124-136, 2010.

2. Klaassen I, Van Noorden CJ and Schlingemann RO: Molecular basis of the inner blood-retinal barrier and its breakdown in diabetic macular edema and other pathological conditions. Prog Retin Eye Res 34: 19-48, 2013.

3. Leto G, Pricci F, Amadio L, Iacobini C, Cordone S, Diaz-Horta O, Romeo G, Barsotti P, Rotella CM, di Mario U and Pugliese G: Increased retinal endothelial cell monolayer permeability induced by the diabetic milieu: Role of advanced non-enzymatic glycation and polyol pathway activation. Diabetes Metab Res Rev 17: 448-458, 2001.

4. Segawa Y, Shirao Y, Yamagishi S, Higashide T, Kobayashi M, Katsuno K, Iyobe A, Harada H, Sato F, Miyata H, et al: Upregulation of retinal vascular endothelial growth factor mRNAs in spontaneously diabetic rats without ophthalmoscopic retinopathy. A possible participation of advanced glycation end products in the development of the early phase of diabetic retinopathy. Ophthalmic Res 30: 333-339, 1998.

5. Murata T, Nagai R, Ishibashi T, Inomuta H, Ikeda K and Horiuchi S: The relationship between accumulation of advanced glycation end products and expression of vascular endothelial growth factor in human diabetic retinas. Diabetologia 40: 764-769, 1997.

6. Lu M, Kuroki M, Amano S, Tolentino M, Keough K, Kim I, Bucala R and Adamis AP: Advanced glycation end products increase retinal vascular endothelial growth factor expression. J Clin Invest 101: 1219-1224, 1998.

7. Antonetti DA, Barber AJ, Khin S, Lieth E, Tarbell JM and Gardner TW: Vascular permeability in experimental diabetes is associated with reduced endothelial occludin content: Vascular endothelial growth factor decreases occludin in retinal endothelial cells. Penn State Retina Research Group. Diabetes 47: 1953-1959, 1998

8. Kim J, Lee YM, Kim CS, Sohn E, Jo K, Shin SD and Kim JS: Ethyl pyruvate prevents methyglyoxal-induced retinal vascular injury in rats. J Diabetes Res 2013: 460820, 2013.

9. Hsieh CF and Chaw SM: Osteomeles schwerinae C. K. Schneid. (Rosaceae): A new record for the flora of Taiwan. Bot Bull Acad Sin 37: 281-285, 1996.

10. Lee J, Jang DS, Yoo NH, Lee YM, Kim JH and Kim JS: Single-step separation of bioactive flavonol glucosides from Osteomeles schwerinae by high-speed counter-current chromatography. J Sep Sci 33: 582-586, 2010.

11. Kim JS, Kim J, Kim CS, Kim YS, Shon E, Jung D, Lee YM, Jung SH and Lee YR: Phenyl derivatives or pharmaceutically acceptable salts thereof, method for preparing same, and composition comprising same as active ingredients for preventing, improving or treating diseases related to vascular endothelial cells or diabetes. Patents, 2013.

12. Vlassara H, Striker LJ, Teichberg S, Fuh H, Li YM and Steffes M: Advanced glycation end products induce glomerular sclerosis and albuminuria in normal rats. Proc Natl Acad Sci USA 91: 11704-11708, 1994.

13. Kim J, Kim KM, Kim CS, Sohn E, Lee YM, Jo K and Kim JS: Puerarin inhibits the retinal pericyte apoptosis induced by advanced glycation end products in vitro and in vivo by inhibiting NADPH oxidase-related oxidative stress. Free Radic Biol Med 53: 357-365, 2012.
14. Sander B, Larsen M, Engler C, Lund-Andersen $H$ and Parving HH: Early changes in diabetic retinopathy: Capillary loss and blood-retina barrier permeability in relation to metabolic control. Acta Ophthalmol (Copenh) 72: 553-559, 1994.

15. Giebel SJ, Menicucci G, McGuire PG and Das A: Matrix metalloproteinases in early diabetic retinopathy and their role in alteration of the blood-retinal barrier. Lab Invest 85: 597-607, 2005.

16. Stitt AW: Advanced glycation: An important pathological event in diabetic and age related ocular disease. $\mathrm{Br} \mathrm{J}$ Ophthalmol 85: 746-753, 2001.

17. Kim J, Kim CS, Sohn E, Lee YM, Jo K, Shin SD and Kim JS: Aminoguanidine protects against apoptosis of retinal ganglion cells in Zucker diabetic fatty rats. Eur Rev Med Pharmacol Sci 18: 1573-1578, 2014.

18. Thornalley PJ: Use of aminoguanidine (Pimagedine) to prevent the formation of advanced glycation endproducts. Arch Biochem Biophys 419: 31-40, 2003

19. Yoon J, Lee H, Chang HB, Choi H, Kim YS, Rho YK, Seong S, Choi DH, Park D and Ku B: DW1029 M, a novel botanical drug candidate, inhibits advanced glycation end-product formation, rat lens aldose reductase activity and TGF- $\beta 1$ signaling. Am J Physiol Renal Physiol 306: F1161-F1170, 2014.

20. Kim J, Jeong IH, Kim CS, Lee YM, Kim JM and Kim JS: Chlorogenic acid inhibits the formation of advanced glycation end products and associated protein cross-linking. Arch Pharm Res 34: 495-500, 2011.

21. Yan H, Guo Y, Zhang J, Ding Z, Ha W and Harding JJ: Effect of carnosine, aminoguanidine and aspirin drops on the prevention of cataracts in diabetic rats. Mol Vis 14: 2282-2291, 2008.

22. Makita Z, Vlassara H, Cerami A and Bucala R: Immunochemical detection of advanced glycosylation end products in vivo. J Biol Chem 267: 5133-5138, 1992.

23. Stitt AW, Bhaduri T, McMullen CB, Gardiner TA and Archer DB: Advanced glycation end products induce blood-retinal barrier dysfunction in normoglycemic rats. Mol Cell Biol Res Commun 3: 380-388, 2000.

24. Vlassara H, Fuh H, Makita Z, Krungkrai S, Cerami A and Bucala R: Exogenous advanced glycosylation end products induce complex vascular dysfunction in normal animals: A model for diabetic and aging complications. Proc Natl Acad Sci USA 89: 12043-12047, 1992.

25. Wautier JL, Zoukourian C, Chappey O, Wautier MP Guillausseau PJ, Cao R, Hori O, Stern D and Schmidt AM: Receptor-mediated endothelial cell dysfunction in diabetic vasculopathy. Soluble receptor for advanced glycation end products blocks hyperpermeability in diabetic rats. J Clin Invest 97: 238-243, 1996.

26. Xu HZ and Le YZ: Significance of outer blood-retina barrier breakdown in diabetes and ischemia. Invest Ophthalmol Vis Sci 52: 2160-2164, 2011

27. Sheikpranbabu S, Kalishwaralal K, Lee KJ, Vaidyanathan R, Eom SH and Gurunathan S: The inhibition of advanced glycation end-products-induced retinal vascular permeability by silver nanoparticles. Biomaterials 31: 2260-2271, 2010.

28. Furuse M, Hirase T, Itoh M, Nagafuchi A, Yonemura S, Tsukita S and Tsukita S: Occludin: A novel integral membrane protein localizing at tight junctions. J Cell Biol 123: 1777-1788, 1993.

29. Bucolo C, Ward KW, Mazzon E, Cuzzocrea S and Drago F: Protective effects of a coumarin derivative in diabetic rats. Invest Ophthalmol Vis Sci 50: 3846-3852, 2009.

30. Kim J, Kim CS, Lee IS, Lee YM, Sohn E, Jo K, Kim JH and Kim JS: Extract of Litsea japonica ameliorates blood-retinal barrier breakdown in db/db mice. Endocrine 46: 462-469, 2014.

31. Schulzke JD, Gitter AH, Mankertz J, Spiegel S, Seidler U, Amasheh S, Saitou M, Tsukita S and Fromm M: Epithelial transport and barrier function in occludin-deficient mice. Biochim Biophys Acta 1669: 34-42, 2005. 